\title{
DERECHOS HUMANOS Y GÉNERO
}

\author{
Rita Radl PhilipP*
}

\begin{abstract}
RESUMEN: Esta exposición trata el tema de los derechos humanos y género como un problema central en el campo de las investigaciones de derechos humanos y educación. Comenzamos por una discusión de los conceptos sexo-género. En el segundo punto, enfocamos el tema del concepto de persona y la dignidad humana subrayando su origen en el pensamiento cristiano. Esta tarea sigue con una clarificación del tema del movimiento feminista por los derechos de las mujeres. En la tercera parte indagamos en relación con nuestro tema la contribución de las cuatro conferencias mundiales referentes a las mujeres. Concluimos con unas reflexiones sobre la importancia de una educación basada en un "a priori" universal en cuanto a la diferencia e igualdad de género.
\end{abstract}

Palabras clave: Sexo-género. Derechos humanos. Movimiento feminista. Conferencias mundiales de las mujeres. Educación.

\section{HUMAN RIGHTS AND GENDER}

ABSTRACT: This paper approaches the theme of human rights and gender as a central problem in the field of human rights and education. It begins by a discussion of sex-and gender. Next, it focuses on the concepts of person and human dignity, stressing that they originated in Christian thought. It then elucidates the issue of the feminist movement for women's rights. Its third part questions the contribution of the four World Conferences on Women organized by the United Nations. It then concludes with a few reflections on

Doctora en Filosofía y Ciencias de la Educación e directora del Centro Interdisciplinario de Investigacións Feministas e de Estudos de Xénero (CIFEX), Universidad de Santiago de Compostela. E-mail: ritam.radl@usc.es 
the importance of an education based on a universal "a priori" as for gender differences and gender equality.

Key words: Sex-gender. Human rights. Feminist movement. World Conferences on Women. Education.

\section{Introducción}

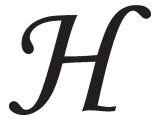

a sido ciertamente un camino largo y arduo que la historia de la humanidad ha tenido que labrarse a lo largo de muchos siglos para llegar a la formulación de unos derechos que se desprenden mismamente de la propia condición humana para todos los seres humanos. Aún cuando, tal y como confirman lamentablemente muchos informes de organismos humanitarios internacionales (Amnesty Internacional etc.), en la actualidad, en absoluto, se ha hecho realidad efectiva la aplicación de unos derechos a todos los seres humanos, una concepción mínima de los derechos humanos independientemente de su condición particular a todos los sujetos que viven en nuestro planeta. Quienes de forma más flagrante y sistemática se ven constantemente privados de sus derechos de poder desarrollarse libremente como personas en todos los rincones de nuestro mundo son indudablemente las mujeres, las mujeres como colectivo que en todas las sociedades particulares constituyen de forma natural como mínimo el $50 \%$ de la población de éstas. A las féminas les es negado moverse libremente en las sociedades, se les coacciona y se les obliga vestirse de una forma determinada, en muchas sociedades no tienen voz ni voto, no pueden muchas veces ni siquiera ganarse su propio sustento, se les obliga a casarse y someterse a la voluntad de una familia y marido que les maltrata, se les mantiene en la pobreza y dependencia absoluta, se les niega recibir una educación mínima, se les impide ejercer su profesión, se les viola impunemente en cualquier conflicto bélico y se les niega el ejercicio de sus derechos de ciudadanía. Hablar de los derechos humanos en un tomo especialmente dedicado a ello requiere, ciertamente, una visión especial referente a las mujeres, a su condición de género, y más si cabe, en un espacio específico que suscita la temática de la educación humana. Por este motivo se impone, casi por sí solo, dedicar este articulo a la cuestión de los derechos humanos y género.

A continuación, comenzaremos por una aclaración conceptual del vocablo "género", para centrarnos, en el siguiente punto, en la dignidad 
humana y el concepto de persona introducido en el contexto sociohistórico de nuestras sociedades por la concepción cristiana, abundando en su significado desde una perspectiva feminista y del género. El apartado tres se ocupa del movimiento histórico por los derechos de las mujeres y del movimiento feminista moderno. Acto seguido analizaremos las conferencias mundiales de las mujeres. Concluiremos con unas reflexiones sobre la igualdad y diferencia de los géneros en la educación.

\section{Aclaración conceptual sexo-género}

A partir de los años 80 se introduce en el ámbito de los estudios de las mujeres un uso diferencial de los conceptos sexo-género, si bien, tal y como refiere Izquierdo (1998) la utilización del concepto género es anterior. Ya en los años 60 Robert Stoller y John Money, en el campo de las terapias médico-psicológicas, se percatan en sus sesiones clínicas de una diferenciación importante en relación con la identidad sexual de sus pacientes en cuanto a su pertenencia de sexo y sus sentimientos reales en relación a la misma, esto es, que la autoconcepción identitaria no tiene porque ser la correspondiente a la clasificación biológico-corporal del sujeto. A partir de este momento Money (1966) propone disociar la identidad del sujeto en tanto que persona - su rol de género (gender rol) - de su pertenencia de sexo - su rol de sexo (sex role). Este autor ya llega a afirmar que la identidad de género corresponde a un proceso de elaboración social, mientras que la identificación basada en las diferencias biológico-hormonales nos clasifica como personas pertenecientes a un sexo u otro. En el debate de los women's studies se adopta, a partir de los años 80, el concepto género precisamente para subrayar la importancia del aspecto constructivo social en cuanto a las identidades de las mujeres y varones, sus funciones y roles sociales.

El concepto sexo, aún cuando representa un término ambiguo, permite tanto una lectura biológico-determinista como una más antropológico-cultural, se refiere de forma restrictiva a una categoría biológica. En este sentido alude a las diferencias anatómico-fisiológicas entre varones y mujeres, que como tales apuntan también biológicamente a un morfismo, a una diversidad. Es más, la definición de lo sexual tiene en sí ya claramente tintes culturales e incorpora interpretaciones en este sentido. Sin embargo, hay una base biológico-fisiológica clara de las 
diferencias que permanecen ciertamente con el tiempo. Precisamente por ello, y para subrayar que en las conductas efectivas de mujeres y hombres a parte de los aspectos propiamente individuales intervienen además de modo explícito elementos culturales y sociales histórico concretos, distinguimos hoy el concepto género. Cabe destacar que sin embargo, no han sido pocas las voces críticas con esta diferenciación conceptual. Así, por ejemplo, resalta Haraway (1996), como los términos sexo/género parten realmente de un sistema de binomios en cuanto a los constructos naturaleza-cultura, naturaleza-historia, lo natural-humano, recurso-producto etc. Linda Nicholson (1994) pone de relieve el hecho de que a pesar de que la diferenciación sexo-género recoge la distinción entre lo biológico y lo cultural, la interrelación entre ambas categorías hay que verla realmente como un "continuum".

Ahora bien, en términos sociológicos los constructos conceptuales sexo-género aluden a una contraposición analitica esencial, absolutamente importante para afrontar la explicación del proceso de cambio social, que es la de una construcción biológico-fisiológica versus una construcción sociocultural. Desde nuestro punto de vista subrayamos, especialmente, que la conducta de género - el comportamiento femenino y masculino en tanto que rol social - corresponde a un proceso de construcción social y como tal apunta a las características, esto es, a las "diferencias psicológicas, sociales y culturales entre los hombres y mujeres", haciéndonos eco de las palabras de Giddens (1994, p. 196). Las diferencias de género tienen como soporte las diferencias biológicas, anatómicas y fisiológicas, es decir, las sexuales, pero siempre van más allá de estas últimas. De modo preciso emergen de las participaciones, experiencias y vivencias sociales activas de los sujetos a base de las diferencias sexuales, y desde un punto de vista sociológico incluyen las experiencias sociales, culturales y psicológicas de los sujetos, por cuyo motivo el comportamiento y la identidad de género de una persona no son pensables sin su pertenencia de sexo.

Una concepción de este tipo en lo que respecta a las identidades de género deja claro dos aspectos fundamentales para nuestro tema: en primer lugar, que los rasgos específicos de los roles femenino y masculino dependen, a su vez, de las experiencias históricas y sociales, colectivas y personales, de las personas y, en segundo lugar, que éstas incorporan modificaciones y cambios. Por este motivo el constructo conceptual género alude, a su vez, a la constelación histórico-social de las 
relaciones intergénero que siempre muestran las constelaciones reales y redes de poder que atañen también al tema de los derechos humanos y su significado en función del género. Dicho en otras palabras, la aplicación de la categoría género a nuestro contexto insiste en una lectura crítica y crítico-ideológica de las relaciones entre mujeres y varones y rechaza una conceptualización descriptivo-neutral del término, a pesar de que en la actualidad el vocablo género se ha convertido, al menos en parte, en un término científico axiológico neutral al uso que ha perdido su noción específico-feminista. En resumen, en el ámbito de las Ciencias Humanas y Sociales tratamos del género, y en nuestro contexto mantenemos una noción crítico-ideológico-feminista.

\section{La dignidad humana y el concepto de "persona"}

La visión de que todos los sujetos son portadores de una misma dignidad que, a su vez, presupone a la persona como sujeto de derecho y de derechos y, de facto, la perspectiva de que todos los sujetos, por si mismos, independientemente de su pertenencia de clase, condición social etc. cuentan con una dignidad en tanto que seres humanos que son, una dignidad que es incompatible con un trato y una visión clasista de las personas, tiene una historia todavía bien reciente y, en absoluto, es hoy día aún una realidad en muchos sitios del mundo. Se han sucedido imperios y culturas que en el contexto global de nuestro planeta constituyen un referente, como es el caso de los imperios griego y romano, por ejemplo, en cuyos largos gobiernos solo ha podido observarse un muy restringido respeto por unos derechos de las personas que además se aplicaban únicamente a determinados sujetos. Concretamente, en la sociedad griega se dirigían solo a los varones de la clase dirigente, a los comerciantes, intelectuales y soldados que participaban en el "ágora" como espacio público donde se determinaban cuestiones relevantes de la convivencia. Si bien es cierto que en el orden social de la Grecia Clásica se identifican unos primeros elementos de participación democrática, éstos solo tienen validez para los varones de los estamentos sociales altos. Cabe pues preguntarse cuándo nace la idea de la dignidad humana, cuándo da comienzo la idea de unos derechos y una dignidad que son inherentes a todas las personas. Sobre este particular hemos de remontarnos al ideario cristiano y, por tanto, a los origenes del cristianismo. Asi es un hecho que el tema de los derechos y 
la dignidad humana para todos los seres humanos, la persona como sujeto de derechos y de derecho, está presente en la historia occidental desde los comienzos del cristianismo, aún cuando este particular es olvidado a menudo en foros científico-sociales que pretenden una reflexión sobre el ser humano y la adecuación de las circunstancias sociales a su condición. Es la concepción cristiana del ser humano, la que le concede a todos los seres humanos la misma condición de dignidad humana, la que elimina la visión clasista del ser humano y de sus derechos. En el seno de la filosofía y conocimiento griegos y en la sociedad romana la concepción del ser humano como portador de derechos se aplica, tal y como mencionamos con anterioridad, solo a determinados sujetos, a aquellos que tienen la condición de "ciudadano": los hombres-varones del estamento social alto o medio-alto que son poseedores de propiedades o títulos, mercaderes, a veces sabios y los soldados. Quedan excluidos de esta condición las mujeres, esclavas, esclavos y, por supuesto, las niñas y niños.

De modo preciso, aparece la formulación la dignidad humana y, por tanto, de los derechos de las personas para todos los seres humanos de forma explícita como idea principal en el cristianismo como presupuesto básico. Todos los seres humanos, esto es, hombres, mujeres, niños, esclavos, son iguales, son potencialmente seres de Dios. Regine Pernoud subraya que San Pablo había dicho: "Ya no hay griego ni judío, ni hombre, ni mujer" lo que en adelante cuenta es la "persona" Pernoud (1987, p. 28). El mismo vocablo "persona" es introducido en el pensamiento cristiano que aporta su significación. Hasta este momento el término "persona" no tenía la connotación que manejamos hoy. Era la mascara que se usaba en el teatro y que identificaba al personaje. "A partir de entonces el término se carga con una significación nueva que responde a una realidad nueva” (ibíd, p. 28 y ss ). Así, es un fenómeno especialmente destacado una y otra vez, que las mujeres y los esclavos son los primeros que abrazan la nueva creencia religiosa. Y este extremo se explica por el hecho de que se les consideraba igual y con los mismos derechos que los varones de la clase ciudadana, eran iguales ante los ojos de Dios. La autora citada ha investigado y demostrado minuciosamente el papel de las mujeres en la primera etapa del cristianismo y en el proceso de la creación de las congregaciones femeninas a partir del siglo v. Demuestra como "la primera en beneficiarse será la mujer". Se fundan, entonces, las congregaciones femeninas que se convierten en el primer 
espacio de dominio femenino exclusivo, dedicado a la producción y reproducción de cultura (estudio y copia de la Biblia, la instrucción de otras mujeres y de los niños de las clases nobles). Las mujeres pueden, por primera vez, decidir libremente su vida. Entre otras cosas se les pregunta, si han venido libremente para contraer matrimonio. Además es digno de mención que las reglas de las órdenes las aprobaban en esta época exclusivamente sus miembros que eran potencialmente todos iguales. Existe realmente una "democracia participativa", los miembros de las congregaciones eligen a sus superioras. La superiora es la máxima autoridad de los conventos y el Papa de Roma, como cabeza de la Iglesia Católica, no interviene en la aprobación de las reglas de las congregaciones femeninas hasta finales de la Edad Media, cuando se impone cada vez más el principio patriarcal basado a su vez en el derecho romano, copiado y adaptado por el derecho eclesial.

Ahora bien, también es cierto que esta concepción cristiana de la persona, aplicada a las mujeres, esclavos etc., no se asienta de forma firme ni siquiera en el seno de la institución que sigue las huellas del mensaje cristiano, la Iglesia Católica, que cae de facto cada vez más en las redes de una concepción "precristiana" de la mujer a través de la recepción y adaptación paulatina del derecho romano. Es más, la conceptualización del ser humano, apoyada en la interpretación jurídicojudeo-romana antifeminista y de tintes patriarcales y androcéntricos, se convierte en el pensamiento de la ilustración, en el modelo clásico para la reclamación de la libertad y racionalidad del sujeto, excluyendo a las mujeres de forma sistemática de esta condición, del saber y de la vida pública. No obstante, es en este instante, cuando, por otra parte, se inicia un movimiento de vindicaciones por los derechos de las mujeres que ya no ha de pararse jamás, un movimiento que conecta, no obstante, con escritos muy anteriores que precisamente reclaman el legado de esta concepción cristiana inicial y revolucionaria desde el punto de vista de los derechos humanos referente a las mujeres como es el caso de la obra de Christine de Pizan, o de Gertrud von Bingen, ${ }^{1}$ de obligada referencia en el contexto que nos concierne.

\section{El movimiento feminista y los derechos de las mujeres}

Los origenes del feminismo como movimiento social de vindicaciones tienen su arraigo, efectivamente, en el siglo XVIII, más 
concretamente al final del siglo XVIII, y la relación de sus tesis principales con el ideario central de la ilustración es innegable, remontándose, tal y como habíamos dicho, a la concepción de la dignidad humana para todos los seres humanos, la persona como sujeto de derechos y de derecho, del cristianismo, pero ya mezclando los elementos claramente patriarcales de la concepción del ser humano, procedente del derecho romano y de la tradición judeo-hebrea.

Desde un ángulo político-social, el feminismo, en tanto que movimiento social y político de las vindicaciones de igualdad para las mujeres, conecta de modo preciso con dos corrientes ideológicas, a saber: con la Revolución Francesa, por una parte, y con el movimiento socialista obrero, por otra.

Como movimiento basado en los ideales de la Revolución Francesa el feminismo entronca de modo evidente con los contenidos centrales de las vindicaciones de esta Revolución que reclama los valores de "liberte"", "egalite" y "fraternite" para todos, pero refiriéndose a aquellos que eran poseedores de propiedades, los burgueses. Desde un punto de vista de pensamiento social, la Revolución Francesa se basa en los valores defendidos por los grandes pensadores de la ilustración (Kant, Hume, Bacon, Descartes etc.). Éstos partían de la base de que el ser humano es ante todo un ser libre y racional, que como tal puede llegar a conocer y organizar a fondo su mundo haciendo uso de su raciocinio, de sus facultades intelectuales y de las evidencias empíricas.

En cuanto al vínculo del feminismo con el movimiento basado en el pensamiento socialista obrero, es preciso destacar que en el seno de las explicaciones teórico socialistas, la existencia humana, la vida humana y el significado de las instituciones sociales y políticas diversas se consideran en conexión con las condiciones de explotación que sufre la clase obrera en el capitalismo. La vida de las mujeres está determinada por su pertenencia de clase y ésta es más fuerte que la condición de género. No obstante, Friedrich Engels (2008) introduce ya una perspectiva particularmente interesante en cuanto a la condición de género cuando argumenta que las mujeres constituyen la "clase proletaria" frente a los varones que representan la "clase burguesa" en el seno de la familia burguesa de rasgos patriarcales, si bien estas ideas tienen más un significado teórico que político-práctico. 
La idea de la igualdad de la mujer aparece formulada explícitamente a finales del siglo XVIII en el contexto francés e inglés. Olympe de Gouges la introduce en 1791 en su Declaración de los derechos de la mujer como ciudadana, en el contexto francés, aún cuando para ella ha tenido unas consecuencias especialmente trágicas ya que le comporta su muerte en la guillotina. Pero el caso de ella muestra como en el propio movimiento revolucionario francés la idea de la igualdad de los derechos ciudadanos para las mujeres ya está presente, si bien tímidamente, a pesar de que en el mismo momento de la revolución esta abocada al fracaso. Por otra parte, Mary Wollstonecraft defiende en 1792 desde Inglaterra en su Vindicación de los derechos de la mujer que "la mujer tiene el mismo derecho que el hombre a la educación". Esta autora contradice de forma concienzuda y brillante los argumentos que Rousseau expone en su obra Emilio, negando a las mujeres una educación basada en la libertad, autonomía, creatividad y racionalidad, en realidad, su condición de sujeto. Este teórico, supuestamente el padre de la pedagogía moderna centrada en el "niño", contempla el derecho de poder desarrollarse libre y autónomamente como ser humano solo para el varón Emilio, las mujeres quedan privadas de este derecho humano. Las pensadoras citadas enlazan con las aportaciones de otras muchas mujeres que ya habían reclamado para el colectivo femenino el derecho de ser consideradas "persona" igualmente que los varones, como es el caso de Gertrud von Bingen en el siglo x y el de Chistine de Pizán en el XV, ya antes mencionadas.

Desde un punto de vista político, a partir de la argumentación de Wollstonecraft se va abriendo lentamente el camino al reconocimiento de los derechos de dignidad humana y de ciudadanía para las mujeres, esto es, el derecho a la educación y la participación política, tal y como lo entendemos hoy día. La reclamación de los derechos de las mujeres está desde los principios centrada en el derecho a la educación, una educación en condiciones de igualdad, conservando algunos aspectos particulares referidos a temas específicos de la feminidad, y el acceso de las mujeres a la educación superior, objetivo, este último, especialmente relevante en el contexto alemán, inglés y español.

Otro tema esencial de las vindicaciones de las mujeres gira en torno a los derechos de ciudadanía. Aquí es especialmente relevante el reconocimiento del derecho al voto, cuestión que centra las vindicaciones feministas sobre todo a finales del siglo XIX en Inglaterra, EEUU 
y Alemania, y a principios del siglo xx en el contexto de otros países europeos y de Sudamérica, como es el caso de Brasil. La consecución del voto político para las mujeres es un logro fundamental que promueve el movimiento alrededor del sufragio femenino.

Un punto y aparte referente a la lucha por los derechos de las mujeres lo constituye el final de la Segunda Guerra mundial. A partir de este periodo, cambia sustancialmente la situación social y política y aparece una de las obras fundamentales del feminismo moderno que ha influenciado, asentado y afianzado las bases del pensamiento feminista contemporáneo no solo en Europa. Estamos hablando de la obra El segundo sexo, de Simóne de Beauvoir (1999). Este libro defiende, con especial ímpetu, la idea de una mujer independiente, libre y "no dividida", argumenta a favor de una mujer "sujeto", cuya vida y destino no dependan de una relación de "alteridad", que la convierte en mero yo relacional. Realizando un agudo análisis de la concepción de la mujer en la civilización occidental desde ópticas teóricas diversas, concluye que todas las disciplinas científicas mantienen el postulado de la inferioridad de la mujer. Es la obra clave para entender el movimiento feminista moderno de la época de la postguerra.

Simone de Beauvoir reclama expresamente la igualdad de las mujeres en aquel ámbito que esta ligado al estatus de la superioridad del hombre, que es el espacio público y laboral extradoméstico La recepción de las ideas de Simone de Beauvoir y la incidencia de las mismas en el movimiento moderno por los derechos de las mujeres se efectúa a partir de los años 60. Muchas mujeres feministas (Betty Friedan, Alice Schwarzer, Judith Mitchell, Kate Millet, etc.) denuncian la existencia de un sistema que impide la aplicación de los derechos, ya constitucionales, a las mujeres en los sistemas democrático-constitucionales modernos, situación que lleva además a la implementación de una perspectiva feminista propia en el contexto académico que conduce finalmente a la construcción del campo de los women's y gender studies. Estos estudios como investigaciones comprometidas con la causa de las mujeres ayudan en la clarificación aún más profunda de la situación de las mujeres que además abren el camino a eventos políticos que inciden en una mayor eficacia del proceso de la consecución de los derechos para todas las mujeres a nivel mundial. No podemos obviar en nuestro contexto el papel crucial que han tenido las cuatro conferencias 
mundiales sobre las mujeres convocadas por parte de las Naciones Unidas en el proceso de la aplicación de los derechos humanos al colectivo de las mujeres.

\section{Las conferencias mundiales de las mujeres}

El movimiento feminista moderno, a parte de lograr la aplicación de los principios del derecho a la situación jurídica de las mujeres, particularmente en el contexto europeo y americano, ha conseguido, ante todo, una mayor sensibilización en relación con los derechos de las mujeres en el seno de algunos organismos oficiales en el contexto internacional, como es el caso de las Naciones Unidas. Concretamente a partir de 1975 este organismo ha convocado cuatro conferencias mundiales sobre las mujeres, que han incidido, indudablemente, en poner en el centro del debate los derechos de las mujeres, o bien, la extensión explícita del principio de la igualdad de los derechos y los derechos de la dignidad humana a todas las mujeres.

Las cuatro conferencias mundiales convocadas por las Naciones Unidas en el último cuarto del siglo XX han contribuido a situar la causa de la igualdad de las mujeres en el mismo centro del temario mundial. Las conferencias han unido a la comunidad internacional en apoyo de un conjunto de objetivos comunes, con un plan de acción para el avance de las mujeres en todas partes y en todas las esferas de la vida pública y privada.

La primera Conferencia Mundial sobre la condición jurídica y social de ls mujeres se convocó en México para que coincidiera con el Año Internacional de la Mujer, en 1975. La Conferencia, conjuntamente con el Decenio de las Naciones Unidas para la Mujer (19761985) proclamado por la Asamblea General cinco meses después a instancias de la Conferencia, inició una nueva era de esfuerzos a escala mundial para promover un cambio ante la situación social de las mujeres y abrir un diálogo de alcance mundial sobre la igualdad de género. Comenzó así un proceso que comprendería las deliberaciones, la negociación, el establecimiento de objetivos, la identificación de obstáculos y el examen del progreso alcanzado.

La Conferencia marcó un hito en la forma en la que eran percibidas las mujeres. Si anteriormente se había considerado que la mujer era 
una receptora pasiva de apoyo y de asistencia, ahora se la veía como asociada plena y en pie de igualdad con el hombre, con los mismos derechos a los recursos y a las oportunidades. Una transformación análoga se estaba produciendo en la manera de enfocar el desarrollo, pues si en un inicio se creía que el desarrollo serviría para mejorar la situación de las mujeres, ahora existía el consenso de que el desarrollo no era posible sin su plena participación. Las mujeres, finalmente, eran consideradas sujetos de su situación social.

La Conferencia exhortó a los gobiernos a que formularan estrategias nacionales y establecieran metas y prioridades en sus esfuerzos por fomentar la participación equitativa de las mujeres. Hacia finales del Decenio de las Naciones Unidas para la Mujer, 127 Estados-miembros habían establecido alguna forma de mecanismos nacionales, instituciones encargadas de la promoción de políticas, investigaciones y programas orientados al cambio de la situación social de las mujeres y su participación en el desarrollo.

El consenso generalizado era que se había alcanzado un progreso considerable, cuando representantes de 145 Estados-miembros se reunieron en Copenhague en 1980, en el marco de la segunda Conferencia Mundial sobre las Mujeres, para examinar y evaluar el plan de acción mundial de 1975. Los gobiernos y la comunidad internacional habían logrado acercarse a la consecución de las metas establecidas en México cinco años antes.

Un acontecimiento importante había sido la aprobación de la Convención sobre la eliminación de todas las formas de discriminación contra las mujeres por parte de la Asamblea General, en diciembre de 1979, uno de los instrumentos más poderosos en la lucha por la igualdad de las mujeres. La Convención, que ha sido denominada "la carta de los derechos humanos de la mujer", vincula actualmente jurídicamente a 165 Estados, que han llegado a ser Estados-miembros en la Convención, lo que los obligaba a presentar un informe en el plazo de un año de la ratificación, y posteriormente cada cuatro años, sobre las medidas que han adoptado para eliminar los obstáculos y hacer efectiva la Convención.

Pese a los progresos conseguidos, la Conferencia de Copenhague reconoció que estaban comenzando a surgir señales de disparidad entre los derechos garantizados y la capacidad de las mujeres para ejercer 
esos derechos. Para abordar esa inquietud, la Conferencia estableció tres ámbitos en los cuales era indispensable adoptar medidas concretas con vistas a las metas de igualdad, desarrollo y paz, determinadas por la Conferencia de México. Estas tres áreas eran la igualdad de acceso a la educación, las oportunidades de empleo y servicios adecuados de atención a la salud.

El movimiento a favor de la igualdad de género había alcanzado un reconocimiento mundial cuando se convocó en Nairobi la tercera Conferencia Mundial de las Mujeres, la "Conferencia Mundial para el Examen y la Evaluación de los Logros del Decenio de las Naciones Unidas para la Mujer: Igualdad, Desarrollo y Paz", en 1985. Puesto que al Foro de Organizaciones no Gubernamentales que se celebró paralelamente asistieron 15.000 representantes, se llegó a clasificar incluso a la Conferencia como "el nacimiento del feminismo a escala mundial". El movimiento de las mujeres, dividido por la política mundial y las realidades económicas en la Conferencia de México, había llegado a convertirse en una fuerza internacional unificada bajo la bandera de la igualdad, el desarrollo y la paz.

Como las mejoras en la situación de las mujeres habían sido, en el mejor de los casos, marginales, se busco la adopción de un nuevo enfoque. La Conferencia de Nairobi recibió el mandato de buscar nuevas formas de superar los obstáculos para alcanzar los objetivos del Decenio.

Se identificaron tres categorías básicas de medidas, a saber: medidas constitucionales y jurídicas, medidas referentes a la igualdad en la participación social y medidas en relación con la igualdad en la participación política y en la toma de decisiones.

Sin embargo, no es hasta la próxima conferencia - la cuarta Conferencia Mundial sobre las Mujeres (Beijing, 1995) - cuando se puede hablar del inicio de un nuevo paradigma en la lucha por la igualdad en funciçon del género.

La transformación fundamental que se produjo en Beijing fue la adopción del enfoque de género, de modo que se reconoció que toda la estructura de la sociedad y todas las relaciones entre los hombres y las mujeres en el interior de esa estructura tenían que ser reevaluadas. Únicamente mediante esa reestructuración fundamental de la sociedad y sus instituciones sería posible potenciar plenamente el papel de las mujeres para que éstas ocuparan el lugar que les correspondía como 
participantes en pie de igualdad con el hombre en todos los aspectos de la vida. Este cambio representó una reafirmación firme de que los derechos de las mujeres eran derechos humanos y de que la igualdad entre los géneros era una cuestión de interés universal y de beneficio para todos.

La cuarta Conferencia aprobó por unanimidad la Declaración y la Plataforma de Acción de Beijing, que era en esencia un programa para la potenciación del papel social de las mujeres. En la Plataforma de Acción se identificaron doce esferas concretas de especial preocupación que se consideraba que representaban los principales obstáculos con respecto a la transformación de la situación social de las mujeres y que exigían el desarrollo de medidas concretas por parte de los gobiernos y la sociedad civil, tales como el tema de la salud, la educación, la violencia, la pobreza etc.

En la Conferencia de Beijing se introducía, por fin, una perspectiva transversal, la perspectiva del "mainstreaming de género", es decir, a partir de este momento los gobiernos se comprometían de incluir de manera efectiva una dimensión de género en todas sus políticas, instituciones, procesos de planificación y de toma de decisiones. Esto significaba que, antes de que se aprobaran las decisiones o se ejecutaran los planes, se debería hacer un análisis de sus efectos sobre los hombres y las mujeres, y de las necesidades de éstos. Por ejemplo, en lugar de procurar que el sistema educacional existente fuera cada vez más accesible a la mujer, la incorporación de una perspectiva de género requeriría una reconstrucción del sistema, de manera que se aviniera por igual a las necesidades de los hombres y las mujeres. A partir de esta última Conferencia Mundial de las Mujeres, la atención no se centraba ya únicamente en éstas y su condición jurídica y social, sino que se empieza a adoptar un punto de vista estructural con respecto a la consecución de los derechos para las mujeres. La realización de las mujeres como portadoras de derechos humanos inalienables es incompatible con unas estructuras políticas y sociales androcéntricas y patriarcales, por cuyo motivo la adopción de este punto de vista por parte de los países que forman parte de las Naciones Unidas sitúa por primera vez el tema en el contexto de las propias estructuras políticas, sociales, económicas como las instancias responsables que impiden la realización efectiva de los derechos de persona para las mujeres. 
Ahora bien, cabe preguntarse en este instante si, efectivamente, a partir de las conferencias mundiales se han superado todos los escollos que impedían la organización vital de las mujeres en condiciones de igualdad y la aplicación de los principios de los derechos humanos a las mismas. Tal como afirmamos con anterioridad, la situación de las mujeres a nivel mundial está lejos de poder ser considerada satisfactoria desde el punto de vista de los derechos humanos, incluso en todos los contextos socioculturales. Valorando los esfuerzos políticos de los organismos internacionales y los logros de estas conferencias, parece que las acciones emprendidas desde el ámbito estructural (internacional y nacional) no han podido, de facto, traducirse aún lo suficientemente en un cambio de la situación concreta de las mujeres, lo cual quiere decir que hasta la fecha no se ha conseguido todavía la implicación directa de las personas, sobre todo en el nivel de las relaciones interpersonales. Esta cuestión apunta a los propios sujetos como los elementos decisivos para poder lograr una transformación real de las estructuras sociales y políticas que impiden la consecución de situaciones de igualdad intergénero desde un ángulo de los derechos humanos, esto es, de dignidad de las personas. Y aquí es donde hemos de dirigir nuestra mirada a los procesos de socialización y educación humana con el propósito de dilucidar esta situación.

\section{Educación, igualdad y diferencia de los géneros}

La educación, en tanto que proceso específico de socialización, incide de manera especial en la socialización de los roles de género, es decir, refuerza, perfila y contribuye de forma esencial a la configuración de las identidades de género femenino y masculino. El papel desempeñado por la educación tiene un significado crucial para el tema que nos ocupa aquí. Es la educación, si cabe, en su faceta de transversalidad y en su sentido más holístico, la que tiene una función esencial en el tema de la transmisión de contenidos vinculados a la defensa de los derechos que requiere la dignidad de todas las personas en tanto que defensa de unos valores que atañen a todos los sujetos femeninos y masculinos. Solo unos valores que defienden su realización, independientemente de la situación particular de los sujetos, de su pertenencia de raza, género y clase social, pueden garantizar unos derechos básicos e iguales para todos éstos. Serían los únicos que pueden promover 
unos procesos de modificación de los elementos estructurales que hacen perdurar la verticalidad de las relaciones entre hombres y mujeres en las sociedades. Y es desde esta perspectiva educativa, desde la cual adquiere una relevancia especial plantear el tema de la Igualdad y Diferencia con vistas a las relaciones intergénero.

No cabe duda de que los primeros planteamientos, aquellos que han logrado el reconocimiento y el cambio de la situación legal para las mujeres, su consideración en la esfera laboral, pública y política, han correspondido a las vindicaciones de la igualdad de las mujeres en comparación con los varones. En cambio, es en los años 80 cuando se introducen perspectivas teóricas que insisten de forma especial en el valor propio de la experiencia histórica de las mujeres, criticando fuertemente la asunción del modelo masculino para una nueva identidad femenina. Desde la posición del feminismo de la diferencia se reclama el reconocimiento expreso de los valores del cuidado, atribuidos históricamente de forma exclusiva a las mujeres.

Pues bien, parece que en estos momentos se están asumiendo en muchos campos planteamientos centrados en la diversidad y diferencia. ¿Pero qué sentido tiene y puede tener una educación y una política que se asientan sólo sobre el reconocimiento de las diferencias?

En lo que respecta a nuestro objeto expositivo, la postura del feminismo de la igualdad es la que propone la igualación de las mujeres con los hombres sobre todo en el espacio público. Las primeras tienen que salir del marco doméstico-privado que es el que conlleva su dependencia económica y la perpetuación de su estatus de inferioridad y su situación de explotadas. Sólo la aplicación de los postulados de la Ilustración a las mujeres, a saber, la idea de que las mujeres son sujetos libres y racionales con una función social y política independiente, puede romper aquel círculo que las atrapa en cuanto que seres meramente sexuados, en última instancia determinadas por sus rasgos sexuales específicos, rasgos estos que emanan de la condición biológico-maternal de todas las mujeres.

No obstante, aún cuando podemos suscribir muchos de los argumentos acerca de la relevancia de la racionalidad instrumental que es, de facto, la que defiende la postura del feminismo de la igualdad, no es menos cierto que la asunción exclusiva de la misma para la redefinición de las relaciones de género, tanto en términos teóricos como 
político-prácticos, encierra en realidad una repulsa a la madre, al rol y la función maternal en tanto que asunción específica de la lógica androcéntrica de fondo que subordina la maternidad a la racionalidad instrumental. ${ }^{2}$ Con ello rechaza la identidad histórico-colectiva de las mujeres. Dicho en otras palabras, la postura feminista de la igualdad merced a una asunción ciertamente incuestionada de la validez universal, a la vez que preeminente de la racionalidad instrumental obvia algo absolutamente básico, que es la importancia de la "lógica maternal", esto es, del valor del cuidado para la vida humana. Es este hecho el que explica y lleva con cierta lógica al pensamiento del feminismo de la diferencia. Éste insiste precisamente en el significado de los valores del cuidado que han integrado, de modo preponderante, históricamente la identidad femenina e introduce una visión teórica sustentada en la validez de la lógica que emerge del reconocimiento de la experiencia vital, precisamente de las mujeres. Una lógica de este tipo permite repensar y resituar la experiencia histórica del colectivo femenino y, más concretamente, las vivencias colectivas de las mujeres alrededor de la maternidad y del cuidado humano. Permite, en definitiva, reclamar estos mismos valores como valores humanos esenciales tanto para las féminas como para los varones.

La lógica del cuidado - o, en otras palabras, una lógica de la comunicación $^{3}$ - es la única que tiene en cuenta el ser humano en su condición de sujeto. Sin embargo, la defensa de los valores del cuidado no puede ir encaminada hacia el reconocimiento arbitrario de las diferencias en tanto que diferencias neutrales - que se refieren en nuestro caso a las del género femenino -, o bien, hacia el abandono total de unos valores lógico-universales, identificada ésta con la lógica masculina, ni hacía la sustitución unilateral de la lógica racional-instrumental por los valores históricamente femeninos. Unos nuevos valores humanos, capaces de guiar las relaciones entre sujetos y, por tanto, los derechos humanos - y esto quiere decir, ante todo, de sujetos femeninos y masculinos - han de asentarse en el presupuesto de la universalidad de la razón humana que es la que justifica, a la vez que exige, una "igualdad de condiciones" a partir de la cual es posible reconocer diferencias y diversidades de tipo individual o colectivo. Sin esta base de unas condiciones mínimas, de este "a priori universal", para todas y todos, el reconocimiento de diferencias y diversidades equivale a un relativismo absoluto de valores y normas sociales. Bajo estos últimos 
habría que reconocer en un mismo plano asimismo formas de sometimiento cultural injustificables como es el caso de la negación de la educación, formación y ejercicio profesional de las mujeres en ciertos regímenes islamistas o la práctica de la ablación, de la mutilación genital femenina, o el sueldo inferior de las mujeres en comparación con el de sus compañeros del sexo masculino por el mismo trabajo desempeñado en muchas partes del mundo. Sólo la concepción de una lógica de la comunicación asentada en un a priori universal permite el reconocimiento de las diferencias de género, de modo que éstas redunden efectivamente en unas relaciones de género simétricas $y$, en consecuencia, en la autorrealización de varones y mujeres como sujetos responsables de su propio destino y como portadores de unos derechos inalienables, inherente a la propia condición humana de todas las personas. Es ciertamente esta idea de un a priori universal la que constituye la base de unos derechos humanos que se reconocen en tanto que exigencias mínimas, como consustanciales a la propia existencia humana, a la noción del vocáblo persona, para todos los seres humanos, independientemente de sus circunstancias particulares.

\section{Conclusiones}

Partiendo de unas aclaraciones sobre los conceptos sexo y género, que nos han permitido situarnos en la temática de los derechos humanos y género, hemos profundizado en el presente contexto en el concepto de persona de corte cristiano, que introduce, en definitiva, el tema de la dignidad humana, de unos derechos humanos consustanciales a todas las personas más allá de su condición de sexo, raza etc. No obstante, dos mil años después de la concreción de este pensamiento, aún seguimos debatiendo, reflexionando y esperando la realización de unos derechos humanos para gran parte de más de la mitad del colectivo humano: para las mujeres.

A pesar de los grandes esfuerzos del movimiento feminista y de los acuerdos internacionales alcanzados en las diferentes conferencias mundiales de las mujeres, queda aún un buen trecho para llegar finalmente a la aplicación de unos derechos básicos en pie de igualdad para todas las féminas en este planeta. Todo apunta a que la cuestión más relevante aquí reside en la modificación de las condiciones de la socialización y educación humana. Solo una nueva educación asentada en 
un a priori universal con respecto a las identidades de género permite el establecimiento de unas relaciones intergénero ciertamente más simétricas, y en consecuencia, la autorrealización de todas las mujeres y de todos los varones en tanto que sujetos responsables de su propio destino y como portadores de unos derechos inalienables, inherentes a la propia condición humana de todas las personas. Con todo, esperamos que las diferencias negativas en función del género no perduren en el tiempo. Lo que sí necesitamos, más que nunca, es realizar todos los esfuerzos educativos, políticos, institucionales y personales precisos para lograr este objetivo cuanto antes.

Recebido em fevereiro de 2010 e aprovado em agosto de 2010.

\section{Notas}

1. Véase la obra de Christine de Pizán (1995), de principios del siglo Xv: La ciudad de las damas, y uno de los libros más relevantes de Hildegard de Bingen (2009), original del siglo XII: Libro de las obras divinas (Liber Divinorum Operum). Y sobre su obra: Cirlot (2005), Hildegard von Bingen y la tradición visionaria de occidente.

2. Es un tema que he abordado en otras publicaciones. Remito, especialmente, a Radl Philipp (2001, 2008 y 1992). Véase, asimismo, la crítica concienzuda del concepto androcéntrico de la igualdad por parte de Luce Irigaray, especialmente Irigaray (1992).

3. O de una "racionalidad comunicativa", vocablo introducido por Habermas en 1981 que nosotros hemos aplicado a nuestro contexto. Véase Radl Philipp (2001) y Habermas (1981).

\section{Referencias}

BEAUVOIR, S. El segundo sexo. Buenos Aires: Sudamericana, 1999. $2 \mathrm{v}$.

BINGEN, H. Libro de las obras divinas (Liber Divinorum Operum). Barcelona: Herder, 2009.

CIRLOT, V. Hildegard von Bingen y la tradición visionaria de occidente. Barcelona: Herder, 2005.

ENGELS, F. El origen de la familia, la propiedad privada y el estado. Madrid: Alianza, 2008.

GIDDENS, A. Sociología. Madrid: Alianza, 1994. 
HABERMAS, J. Die Theorie des Kommunikativen Handelns. Frankfurt: Suhrkamp, 1981. 2 v.

HARAWAY, D. Ciencia, cyborgs y mujeres: la reinvención de la naturaleza. Madrid: Cátedra, 1996.

IRIGARAY, L. Yo, tu, nosotras. Valencia: Cátedra, 1992.

IZQUIERDO, M.J. El malestar en la desigualdad. Madrid: Cátedra, 1998

MONEY, J. Sex reassignment as related to hermafroditism. In: BENJamin H. The transsexual phenomenon. New York: Julien, 1966.

NICHOLSON, L. Femimismo/Postmodernismo. Buenos Aires: Feminaria, 1992.

NICHOLSON, L. Interpreting gender. Signs, Chicago, v. 20, n. 1, p. 79105, 1994.

PERNOUD, R. La mujer en la época de las catedrales. Barcelona: Granica, 1987.

PIZÁN, C. La Ciudad de las damas. Madrid: Siruela, 1995.

RADL PHILIPP, R. La nueva definición del rol femenino. In: RADL Philipp, R.; García Negro, M.C. A muller e a súa imaxe. Santiago de Compostela: Universidad de Santiago de Compostela, 1992. p. 29-52.

RADL PHILIPP, R. Acerca del estatus epistemológico crítico de las investigaciones de género. In: RADL PHILIPP, R. (Ed.). Cuestiones actuales de sociología del género. Madrid: CIS-USC, 2001. p. 15-27.

RADL PHILIPP, R. Questôes epistemológicas sobre gênero: o debate atual. Ponta Grossa: Universidade Estadual de Ponta Grossa, 2008.

UNITED NATIONS. Report of the Conference of the International Women's Year. New York: United Nations, 1976.

UNITED NATIONS. Report of the World Conference of the United Nations decade of Women: equality, development and peace. New York: United Nations, 1980. 
UNITED NATIONS. Report of the World Conference to review and appraise the achievements of the United Nations decade for Women: equality, development and peace. New York: United Nations, 1986.

UNITED NATIONS. Report of the Fourth World Conference on Women. New York: United Nations, 1996.

WOLLSTONECRAFFT, M. Vindicación de los derechos de la mujer. Madrid: Debate, 1998. 